\title{
Application de la thérapie EMDR au trouble de la personnalité borderline
}

\author{
Dolores Mosquera \\ Instituto para el estudio del Trauma y los Trastornos de la Personalidad, A Coruña, Espagne
}

Andrew M. Leeds

Sonoma Psychotherapy Training Institute, Santa Rosa, Californie, États-Unis

Anabel Gonzalez

Complejo Hospitalario Universario de A Coruña, Espagne

\begin{abstract}
II y a aujourd'hui un intérêt croissant pour l'utilisation de la thérapie de désensibilisation et de retraitement par les mouvements oculaires (EMDR), au-delà du traitement de l'état de stress post-traumatique (ESPT) où son application est bien établie. Étant donné le fort consensus scientifique actuel autour de la responsabilité des vécus traumatiques du passé dans le développement d'un trouble de la personnalité borderline (TPB), I'EMDR semble en effet pouvoir apporter beaucoup au traitement des personnes qui en souffrent. Cependant, les traits spécifiques de ces patients font que l'application de la thérapie EMDR dans leur traitement s'avérer parfois difficile et nécessite plusieurs adaptations (mineures) des procédures standard de l'EMDR pour l'ESPT. Le présent article propose des principes et des stratégies permettant de préparer de façon sûre et efficace les patients TPB à la thérapie EMDR et d'accéder et de retraiter les origines traumatiques du TPB. Des exemples cliniques sont donnés tout au long de cet article.
\end{abstract}

Mots-clés : trouble de la personnalité borderline ; EMDR ; psychothérapie ; trauma

D ans l'approche psychothérapeutique EMDR (désensibilisation et retraitement par les mouvements oculaires), on considère que les difficultés psychologiques sont principalement causées par l'effet cumulatif d'expériences négatives ou traumatiques non résolues (Shapiro, 2007). Il a été démontré que la thérapie EMDR est un traitement efficace et bien toléré de l'état de stress post-traumatique (ESPT) (Bisson \& Andrew, 2007 ; Foa, Keane, Friedman \& Cohen, 2009 ; Ursano et coll., 2004) et qu'elle est efficace pour traiter aussi bien les ESPT déclenchés à l'âge adulte que ceux qui sont apparus dans l'enfance (AdlerTapia \& Settle, 2009 ; Field \& Cottrell, 2011 ; Korn, 2009 ; Van der Kolk et coll., 2007). En thérapie EMDR, on demande au patient d'observer avec attention ce qui se produit dans ses représentations de perceptions, d'émotions, de sensations, d'actions et de définitions de soi qui sont perturbantes ou dysfonctionnelles, tout en suivant une série de séquences de stimulations bilatérales (SBL) alternées, composées de mouvements oculaires, de tapotements ou de sons alternés (Leeds, 2009 ; Shapiro, 2001). Un large corpus d'études soutient l'hypothèse que le trouble de la personnalité borderline (TPB) est en rapport étroit avec des expériences traumatiques ou défavorables (Ball \& Links, 2009 ; Horesh et coll., 2008 ; Tyrka, Wyche, Kelly, Price \& Carpenter, 2009 ; Zanarini et coll., 2002). Cependant, peu de publications décrivent l'application de l'EMDR chez des patients souffrant de TPB.

Dans deux cas de trouble du stress extrême non autrement spécifié (DESNOS) qui remplissaient également les critères diagnostiques du TPB, Korn et Leeds (2002) ont rapporté la démonstration d'une réduction significative des symptômes après l'étape de développement et d'installation de ressources (DIR) dans la phase de stabilisation du traitement ; cependant, ils

This article originally appeared as Mosquera, D., Leeds, A. M., \& Gonzalez, A. (2014). Application of EMDR Therapy for Borderline Personality Disorder, Journal of EMDR Practice and Research, 8(2), 74-89. Translated by François Mousnier-Lompré. 
n'ont pas évoqué l'utilisation, ensuite, du retraitement EMDR pour traiter les souvenirs traumatiques. Dans un rapport de cas concernant une femme diagnostiquée TPB, Brown et Shapiro (2006) ont rapporté une diminution cliniquement significative des résultats aux sous-échelles du Inventory of Altered Self-Capacities (Briere, 2004), qui sont toutes passées sous le seuil clinique après vingt séances de thérapie EMDR faites sur une période de six mois. La patiente était restée instable dans son fonctionnement conjugal après un traitement initial de dix-huit mois (avec le même clinicien) en psychothérapie cognitive comportementale et psychodynamique/orientée sur la prise de conscience ; deux ans avaient passé, puis la patiente s'était finalement tournée vers une thérapie EMDR.

Le dernier des trois cas rapportés par Wesselmann et Potter (2009) répondait peut-être aux critères du TPB, même si le psychiatre avait diagnostiqué un trouble dépressif majeur chez cette femme. «Mme K. décrivait des signes de dépression sévère, avec de fréquentes idées suicidaires. Elle s'automutilait presque tous les jours, et parfois plusieurs fois par jour, en se scarifiant les bras, les jambes et le ventre avec une lame de rasoir » (p. 187). Ou encore : "Elle était submergée par ses émotions et s'automutilait lorsqu'elle avait eu des contacts avec ses parents "(p. 187). La patiente avait été suivie en thérapie pendant plusieurs années ; elle avait également été internée en psychiatrie pour ses comportements autodestructeurs et avait suivi un an de thérapie comportementale dialectique (TCD ; Linehan, 1993). Mme K. a fait treize séances de thérapie EMDR, intégrées dans quarante séances de thérapie individuelle, sur une période d'un an. Pendant sa thérapie EMDR, elle a continué par ailleurs à participer à des séances hebdomadaires de groupe en TCD. Un an après sa thérapie EMDR, le suivi de cette patiente faisait état d'une rémission complète de ses comportements autodestructeurs et son score à l'Entretien sur l'Attachement chez l'Adulte (AAI, Adult Attachment Interview ; Hesse, 1999) avait évolué de R1 (rejetant) avec une possibilité de NR/D (non résolu/désorganisé) à F1 (sécurité gagnée).

\section{Les facteurs étiologiques du TPB}

\section{Attachement et TPB}

Un lien entre des perturbations précoces de l'attachement et le développement d'un TPB a été postulé par de nombreux auteurs, mais différentes études ont montré que les diverses classifications de l'attachement insécure étaient davantage en rapport avec des traits borderline chez l'adulte. Les discussions concernant ces résultats sont compliquées par la variété des outils de mesure utilisés : certaines études utilisent en effet l'AAI, avec son ensemble dimensionnel de classifications de l'attachement, qui comprend une classification "non résolu / désorganisé »; d'autres recourent à divers instruments d'auto-évaluation présentant des classifications catégorielles totalement différentes, où ne figure pas la classification non résolu/désorganisé. Agrawal, Gunderson, Holmes et Lyons-Ruth (2004) ont passé en revue des études portant sur l'attachement chez des individus répondant aux critères du TPB et conclu que les résultats les plus constants montrent que ces individus ont les types d'attachement non résolu et anxieux. Dans les études utilisant l'AAI examinées par Agrawal et coll., entre $50 \%$ et $80 \%$ des patients TPB étaient classés "non résolus ». En revanche, une étude récente menée par Barone, Fossati et Guiducci (2011), utilisant l'AAI, rapportait que les catégories de type "organisé insécure " (rejetant : $51 \%$ et préoccupé : $35 \%$ ) et " désorganisé " (" non résolu — inclassable »), soit $40 \%$ des sujets, étaient généralement surreprésentées chez les sujets TPB, avec des différences significatives dans la distribution de la classification de l'attachement pour quatre sous-groupes de TPB (co-occurrence de troubles anxieux/troubles de l'humeur, d'abus/ dépendance à une substance, d'abus/dépendance alcoolique et de troubles des conduites alimentaires). Dans les études autoévaluées examinées par Agrawal et coll., qui comprenaient une classification " attachement effrayé ", l'attachement préoccupé était la seconde catégorie la plus fortement rapportée par les sujets TPB. Barone et coll. ont trouvé que l'attachement de type "préoccupé » était le plus prévalent $(52$ $\%$ ) dans le sous-groupe trouble de l'humeur/trouble anxieux. Dans aucune étude globale sur le TPB comprenant la classification préoccupé ou effrayé, l'attachement préoccupé n'était la classification générale la plus prévalente.

\section{Expériences de vie négatives et TPB}

Plusieurs études ont décrit la comorbidité fréquente ESPT/TPB (Driessen et coll., 2002 ; McLean \& Gallop, 2003 ; Pagura et coll., 2010 ; Pietrzak, Goldstein, Southwick \& Grant, 2011). D’autres ont trouvé une relation entre le TPB et des types spécifiques d'abus (Battle et coll., 2004 ; Cohen, Crawford, Johnson \& Kasen, 2005 ; Golier et coll., 2003 ; Goodman \& Yehuda, 2002 ; Grover et coll., 2007 ; Johnson, Cohen, Brown, Smailes \& Bernstein 1999 ; Laporte \& Guttman, 1996 ; Sabo, 1997 ; Tyrka et coll., 2009 ; Yen et coll., 2002 ; Zanarini, 2000 ; Zanarini et coll., 2002). Ball et Links (2009) ont passé en revue la littérature 
portant sur le trauma et le TPB dans le contexte des critères classiques de Hill (1965) permettant d'établir un lien de causalité (force, constance, spécificité, temporalité, gradient biologique, plausibilité, cohérence, preuve expérimentale et analogie). Ces auteurs ont démontré que le trauma peut être considéré, dans un modèle étiologique multifactoriel, comme un facteur causal du développement du TPB.

Une étude préliminaire (Leeds \& Mosquera, 2012) portant sur des patients diagnostiqués TPB a exploré les expériences négatives et traumatiques précoces vécues par ces patients à l'aide de l'Échelle des Expériences familiales au cours de l'Enfance (Family Experiences in Childhood, FECS ; Gonzales, Mosquera \& Leeds, 2011). La FECS est un instrument d'auto-évaluation couvrant les diverses expériences subjectives et traumatiques survenues dans le cadre familial au cours de l'enfance. Ainsi qu'on pouvait s'y attendre, cette étude fait apparaître un ensemble de récits cohérents de vécus négatifs : négligences psychologiques et carences affectives, pertes, inversions des rôles parent-enfant, manques de respect et comportements agressifs. Des expériences traumatiques majeures telles que des agressions sexuelles étaient également très prévalentes $(54 \%$ dans l'échantillon de Gonzales et coll. en 2011), mais les expériences de négligence et de maltraitance se potentialisent probablement entre elles.

Les symptômes du trouble borderline sont similaires aux séquelles courantes et bien connues de la traumatisation précoce (Timmerman \& Emmelkamp, 2001). De nombreux experts ont proposé de classer les symptômes borderline comme des troubles du stress : DESNOS (Van der Kolk, Roth, Pelcovitz, Sunday \& Spinazzola, 2005), état de stress posttraumatique complexe (ESPT-C ; Herman, 1992) et trouble post-traumatique de la personnalité (Classen, Pain, Field \& Woods, 2006). De nombreuses conséquences caractéristiques de la traumatisation précoce, sévère et chronique coïncident avec la symptomatologie du trouble de la personnalité borderline (Driessen et coll., 2002 ; Gunderson \& Sabo, 1993 ; McLean \& Gallop, 2003 ; Roth, Newman, Pelcovitz, van der Kolk et coll., 2005 ; Yen et coll., 2002), indiquant un lien de causalité entre les expériences négatives et traumatiques précoces et l'apparition du TPB chez l'adulte. Dans la mesure où un ensemble de preuves montre que les traumatismes interpersonnels précoces, sévères et chroniques, les perturbations précoces de l'attachement et les expériences négatives contribuent au développement du TPB, il semble évident que l'EMDR a un rôle à jouer auprès des personnes souffrant de TPB.

\section{Génétique et TPB}

Au-delà du lien entre les expériences difficiles et traumatiques du passé et les risques de développement d'un TPB, des facteurs d'ordre génétique et biologique ont été identifiés dans le développement de traits borderline (Siever, Torgersen, Gunderson, Livesley $\&$ Kendler, 2002) ainsi que dans le développement de la sécurité de l'attachement et des capacités de régulation émotionnelle (Brussoni, Jang, Livesley \& MacBeth, 2000 ; Crawford et coll., 2007 ; Goldsmith \& Harman, 1994). La question de savoir dans quelle mesure les facteurs environnementaux et biologiques contribuent au développement du TPB reste sujette à controverse (Bakermans-Kranen-Burg, van IJzendoorn, Bokhorst \& Schuengel, 2004 ; Bokhorst et coll., 2003 ; O’Connor \& Croft, 2001). Le plus probable est que le degré de ces influences varie dans chaque cas et qu'il s'agit d'interactions complexes (Mosquera, Gonzales \& Van der Hart, 2011). En dépit des difficultés actuelles à identifier directement des facteurs génétiques et biologiques chez les personnes souffrant de TPB, ces différents aspects sont pertinents dans une discussion sur la conceptualisation de cas en EMDR et pour l'optimisation d'un traitement pharmacologique, approche parallèle souvent nécessaire chez les sujets TPB. Nous renvoyons le lecteur à Gianoli, Jane, O'Brien et Ralevski (2012) et Lieb, Völlm, Rücker, Timmer \& Stoffers (2010) pour des revues récentes de la pharmacothérapie pour les patients TPB. Ainsi que Shapiro (2010) le fait remarquer, l'EMDR peut être un outil intéressant pour discriminer le rôle de ces expériences précoces dans les difficultés de ces patients.

\section{TPB et dissociation}

Les études indiquent une fréquence élevée de la dissociation pathologique chez les patients TPB (Chu \& Dill, 1991 ; Galletly, 1997 ; Paris \& Zweig-Frank, 1997) avec la présence de symptômes dissociatifs identifiés dans plus de deux tiers des cas de TPB (Korzekwa, Dell \& Pain, 2009 ; Ross, 2007). Quand on utilise l'approche EMDR, il est essentiel d'établir le plus tôt possible la présence éventuelle d'une importante dissociation structurelle (Van der Hart, Nijenhuis \& Steele, 2006), car ces cas nécessitent une " approche progressive " spécifique afin d'identifier et de perlaborer les phobies dissociatives et d'accéder en toute sécurité au matériel traumatique (Gonzales \& Mosquera, 2012). La relation entre dissociation structurelle et TPB est trop complexe pour être développée dans cet article (cf. Mosquera et coll., 2011, pour une revue globale de ce sujet). 


\section{Tenir compte des sous-groupes étiologiques du TPB dans le plan thérapeutique : problèmes dissociatifs, biologiques et d'attachement}

Mosquera et coll. (2011) ont proposé trois sousgroupes de présentations borderline qui sont pertinents pour la conceptualisation des cas en EMDR :

1. Un groupe plus spécifiquement biologique, avec d'importants facteurs biologiques (impulsivité d'origine génétique ou dysrégulation émotionnelle, trouble bipolaire comorbide, ou toxicomanie).

2. Un groupe basé sur l'attachement où prédominent les perturbations précoces de l'attachement.

3. Un groupe dissociatif avec une histoire précoce d'attachement désorganisé et un passé plus grave de traumatisation, et qui présente un trouble dissociatif comorbide ou une prééminence des traits dissociatifs.

Nous aborderons le premier et le second groupe dans cet article. On peut traiter les deux groupes par des procédures similaires, standard, avec les adaptations décrites dans la suite de ce texte. La différence principale est que « le groupe plus biologique » nécessitera une intervention plus orientée sur ces facteurs et sur les conditions comorbides (par exemple, recourir à la pharmacologie pour traiter l'impulsivité ou la dysrégulation émotionnelle, ou pour apporter un soutien spécifique dans les cas d'abus d'alcool ou de drogue). Le troisième groupe - celui qui présente une prééminence des traits dissociatifs - est traité dans la même perspective que les patients souffrant de troubles dissociatifs et, ainsi que le note Shapiro (2001, p. 443), il nécessite des modifications notables des procédures EMDR (voir l'article [non traduit à ce jour à notre connaissance, $\mathrm{NdT}] E M D R$ and Dissociation: The Progressive Approach ; Gonzalez \& Mosquera, 2012). Ces trois groupes ne s'excluent pas mutuellement, mais cette classification peut aider à définir des priorités pour la thérapie et des différences dans la conceptualisation des cas.

Par exemple, une patiente présentant des caractéristiques bipolaires comorbides a nécessité une longue période de stabilisation médicamenteuse, suivie d'une approche progressive de ses principaux souvenirs traumatiques, alternant entre le retraitement EMDR et une psychothérapie plus soutenante lorsque son humeur était déstabilisée en raison de sa vulnérabilité biologique. Chez un autre patient, chez qui prédominaient des symptômes dissociatifs et des hallucinations auditives agressives, il a fallu de longues négociations avec ses différentes parties dissociatives, puis de l'EMDR centré sur ses phobies dissociatives, avant de pouvoir passer au retraitement EMDR de ses souvenirs traumatiques. Néanmoins, il y a un nombre important de patients TPB pour lesquels le retraitement EMDR des souvenirs d'expériences traumatiques peut être proposé rapidement au cours du traitement, et où la gravité des symptômes et les réactions du patient à l'installation du lieu sûr ou à l'IDR ne sont pas des critères essentiels dans le choix du moment opportun pour passer au retraitement des traumas.

\section{TPB et EMDR}

Le modèle du traitement adaptatif de l'information (TAI ; Shapiro, 2001) donne une base conceptuelle pour comprendre comment des informations biographiques portant sur des expériences de l'enfance peuvent être rapportées à des traits spécifiques de la psychopathologie chez l'adulte. Le TAI offre d'autre part un excellent cadre thérapeutique pour le traitement d'un trouble qui, ainsi qu'on l'a mentionné plus haut, est fortement lié aux expériences précoces négatives et traumatiques (Brown $\&$ Shapiro, 2006). Néanmoins, certaines adaptations des procédures standard de l'EMDR sont nécessaires pour travailler avec ce type de patients dans les huit phases de la thérapie EMDR (Leeds \& Mosquera, 2012 ; Mosquera, 2012b ; Mosquera \& Gonzales, 2011a).

Phase 1 de la thérapie EMDR : recueil de I'histoire du patient

Dans l'approche psychothérapeutique propre à l'EMDR, le thérapeute doit rassembler des informations concernant à la fois les problèmes présents et les expériences de vie traumatiques du passé du patient. Toutefois, de nombreux patients TPB ne divulgueront leurs difficultés présentes ou leurs expériences passées les plus en rapport avec leur trouble, qu'à la condition qu'on les aborde avec prudence et de façon particulière. Les informations concernant des patterns d'attachement dysfonctionnels, une toxicomanie, un comportement à risque ou d'autres difficultés peuvent ne pas être révélées spontanément par des patients qui sont souvent plus focalisés sur leurs symptômes anxieux ou dépressifs ou sur leurs difficultés relationnelles du présent. La FECS est un outil précieux pour l'exploration des expériences passées (Gonzales et coll., 2011). La FECS peut servir à explorer des questions dont les patients ne reconnaissent pas le caractère problématique ou dont ils n'osent pas parler en raison d'un sentiment de honte ou d'une conception défensive de «la vie privée de la famille». La FECS va 
bien au-delà d'une simple liste des dix souvenirs les plus traumatisants (Shapiro, 2001), et permet d'explorer un large éventail d'expériences de vie négatives.

Adapter le rythme du recueil de l'histoire à la faible tolérance aux affects du patient. En explorant l'histoire passée et les difficultés actuelles du patient, le thérapeute doit garder à l'esprit les questions de rythme et de tolérance aux affects. Les patients TPB n'ont en effet pas tous la même capacité à gérer les émotions et les souvenirs que fait invariablement remonter l'examen de leurs stratégies d'adaptation déficientes et de leur histoire passée et présente qui est généralement complexe. Par exemple, même si certains patients sont capables d'utiliser par euxmêmes un instrument d'auto-évaluation comme la FECS, beaucoup trouvent perturbante et déstabilisante cette tâche solitaire. Ceux-là ont besoin de remplir le questionnaire de la FECS au cours d'entretiens en commun avec le thérapeute, sur plusieurs séances, avec son soutien et ses conseils. Le thérapeute, lui, doit surveiller de près le niveau d'activation de son patient lors du recueil de son histoire personnelle et se tenir prêt à lever le pied et à dévier le travail vers le développement des capacités de contention, de présentification et de régulation des affects du patient. L'approche standardisée du recueil de ces informations ne convient pas aux patients ayant des histoires complexes de négligence et de trauma. Ce qui peut convenir à un patient peut être totalement inadéquat pour un autre, et le fait de suivre directives et règles ne doit pas faire négliger la situation et les caractéristiques propres à chaque patient. La prise de décision, avec les patients TPB, est plus précise lorsqu'elle est basée sur une relation thérapeutique bien délimitée et, chez le clinicien, sur une bonne connaissance du trouble et du modèle TAI. Ainsi que l'indique Shapiro (2001) : «Il est préférable de fournir aux praticiens un cadre conceptuel ou un modèle qui serve de guide à leur pratique clinique, que de se borner à leur donner une procédure immuable à suivre pas à pas pour utiliser l'EMDR » (p. xiii). Ce point de vue est particulièrement juste avec les patients TPB.

\section{Phase 2 : préparation et stabilisation}

La phase de stabilisation a été décrite comme une étape essentielle avant de passer au travail sur le trauma (Courtois, Ford \& Cloitre, 2009 ; Van der Hart, Brown \& Van der Kolk, 1989). Le traitement du TPB et des troubles du stress extrême précoce et chronique comporte de nombreuses particularités qu'il faut garder à l'esprit, comme le rôle des états mentaux liés à l'attachement et celui des phobies de l'attachement, des affects et des souvenirs traumatiques (Pearlman $\&$ Courtois, 2005 ; Van der Hart et coll., 2006). Le travail avec les patients souffrant de TPB et de trauma complexe est intrinsèquement relationnel, et nécessite souvent de gérer des moments émotionnels intenses et une phobie des affects dans le transfert et le contre-transfert. A la fois avant et pendant le retraitement EMDR des souvenirs traumatiques, il est essentiel de comprendre ces aspects et de disposer de stratégies pour les gérer, pour pouvoir faire ce retraitement de façon efficace et en toute sécurité (Leeds \& Mosquera, 2012).

La relation thérapeutique. L'aspect le plus difficile du traitement des patients TPB est d'établir une relation thérapeutique qui soit à la fois soutenante et bien délimitée avec des patients présentant, à l'âge adulte, d'importantes difficultés sur le plan de l'attachement et des rapports interpersonnels. Il s'agit en particulier de la gestion des fortes réactions émotionnelles qui apparaissent chez le thérapeute durant les séances d'EMDR (Leeds \& Mosquera ; Mosquera, 2012b ; Mosquera \& Gonzales, 2011a, 2011 b). Les cliniciens doivent posséder une tolérance aux affects suffisante pour garder leur capacité de réflexion concernant la signification des fortes émotions qui peuvent surgir en eux lorsque les patients TPB expriment eux-mêmes des émotions violentes (Dworkin, 2005). Ces réactions contre-transférentielles peuvent être des voies d'accès permettant de repérer des questions essentielles qu'il faudra aborder avec habileté, ou les expériences traumatiques passées que le patient, le thérapeute, ou les deux, rejouent sans le vouloir (Dalenberg, 2000). Une formation spécialisée, un entraînement et une supervision sont indiqués lorsqu'on souhaite acquérir ces compétences thérapeutiques. Dans certains cas, les cliniciens devront aborder les origines de leurs propres difficultés de tolérance aux affects par le biais d'une thérapie EMDR personnelle. Dans le processus de création d'une alliance thérapeutique avec un patient TPB, il sera utile au clinicien de se renseigner sur les difficultés que le patient a rencontrées au cours de psychothérapies antérieures, afin de prévenir ou de minimiser les risques de réapparition de ces difficultés relationnelles dans la relation thérapeutique.

Les interventions psychoéducatives. Il faut apporter au patient des éléments de psychoéducation dès le début de la thérapie, concernant les défenses, la résolution de problèmes, les émotions, les besoins et particulièrement le fait de s'occuper de soi-même. Ces informations aideront le patient à comprendre ses difficultés, à prendre de la distance et à développer les ressources dont il aura besoin avant le retraitement des traumas. Ce processus de psychoéducation n'est 
pas forcément de longue durée : en effet, dans certains cas, la meilleure intervention stabilisatrice peut être le traitement, aussi tôt que possible, de souvenirs traumatiques bien choisis. Il ne faut pas considérer la psychoéducation comme une intervention limitée dans le temps, chez les patients TPB, mais comme une succession d'interventions qui accompagneront tout le travail : il sera en effet souvent nécessaire d'apporter des informations adaptées tout au long du processus thérapeutique. Par exemple, des informations sur l'attachement seront introduites en phase 1 au cours du recueil de l'histoire du patient, abordées plus spécifiquement lors du travail concernant le fait de prendre soin de soi, et réintroduites sous forme de tissages cognitifs au cours des phases 4 à 7 .

Travailler sur les patterns de soin personnel. Les patterns de soin envers soi-même sont habituellement problématiques chez les patients TPB. Ceux-ci manquent en effet souvent de bonnes habitudes de prise en charge personnelle et recherchent plutôt une reconnaissance extérieure ou une satisfaction immédiate. Ils éprouvent des difficultés à se protéger et à établir des frontières saines, et ne parviennent pas à trouver un équilibre sain entre prendre soin de soimême et prendre soin des autres. Ils sont souvent incapables de tolérer les affects positifs et ont des difficultés à demander de l'aide : ils sont parfois extrêmement dépendants ou, au contraire, empêchent les autres de leur venir en aide. Nombre d'entre eux se maltraitent en entretenant un dialogue intérieur négatif ou en recourant ouvertement à des comportements autodestructeurs. Dans ce groupe clinique, des procédures spécifiques d'amélioration de ces patterns de soin à soi-même doivent souvent être utilisées lors de la phase de préparation (Gonzales \& Mosquera, 2012 ; Mosquera, 2012a). Dans les cas de trauma complexe, l'étape de la DIR (Korn \& Leeds, 2002 ; Leeds, 2009) devra être élargie pour inclure ces procédures ainsi que le développement de compétences adaptatives spécifiques (Gonzales \& Mosquera, 2012 ; Mosquera, 2004), en donnant la priorité à des cibles qui favorisent un bon fonctionnement dans les activités quotidiennes normales : avoir un sommeil régulier, faire régulièrement de l'exercice, avoir une alimentation ordonnée, s'abstenir de tout abus de substance, avoir des relations sociales et une stabilité financière. On peut faire un «test » pour voir comment les patients réagissent aux affects positifs lors de la DIR, car, dans de rares cas, des réponses paradoxales (négatives) ont été rapportées lors de procédures DIR chez des patients ayant une mauvaise tolérance aux affects positifs (Leeds, 2009, p. 120).
Gérer les pulsions d'automutilation et les risques suicidaires. De toutes les difficultés susceptibles d'être rencontrées lors du traitement des patients TPB, l'automutilation et les menaces de suicide représentent souvent le plus grand défi relationnel pour le thérapeute. Le patient qui s'automutile ou qui pense au suicide crée une situation relationnelle complexe, et l'attention que nous portons, nous autres cliniciens, à nos réactions émotionnelles est un aspect essentiel de la réponse thérapeutique. Le désespoir d'un patient qui ne voit aucun sens à sa vie peut conduire son thérapeute à le considérer comme un cas désespéré. Au contraire, certains thérapeutes peuvent s'impliquer excessivement et se sentir responsables de la vie de leur patient, ce qui mène invariablement le processus thérapeutique à l'impasse. Les automutilations et les menaces suicidaires sont des problèmes complexes, qui peuvent être liés à un manque de régulation émotionnelle, à des patterns négatifs de soin à soi-même, à une culpabilité non traitée ou à des difficultés relationnelles.

La gestion de ces difficultés doit être prise en compte comme un des éléments de la conceptualisation globale du cas, parce qu'avec d'autres patients, les mêmes interventions appliquées au même type de comportements ne seront pas efficaces. Un patient qui menace de se suicider ou qui fait un passage à l'acte suicidaire parce qu'il se sent incapable de gérer les difficultés qu'il rencontre peut réagir mieux à une intervention de type familial, grâce à laquelle il recevra du soutien. Dans d'autres cas de menaces de suicide, au contraire, l'intervention la plus appropriée sera de développer de meilleures procédures de soins à soi-même ou d'installer de meilleures frontières pour réduire la dépendance aux autres et permettre au patient de se concentrer sur ses propres ressources. Un patient ayant tendance à s'automutiler parce qu'il n'a pas de bonnes capacités de régulation pourrait tirer profit de l'approche psychoéducative décrite dans la section suivante, portant sur la régulation émotionnelle. Dans d'autres cas où le comportement d'automutilation du patient est fortement lié à une culpabilité irrationnelle (ex : «C'est ma faute »), l'intervention de stabilisation la plus efficace peut être de retraiter un souvenir central associé à cette cognition négative. Dans ce genre de cas, la cible à retraiter n'est pas le comportement d'automutilation lui-même, mais les circonstances entourant la première occurrence de l'automutilation, l'origine des croyances négatives associées au problème et les souvenirs susceptibles d'être identifiés à travers un pont d'affect, un pont cognitif ou un pont de pulsion (Leeds, 2009 ; Watkins, 1971, 1990).

Comme dans toutes les procédures EMDR utilisées auprès de patients TPB, une relation thérapeutique 
ferme, soutenante, avec des frontières solides, est essentielle dans la gestion de ces situations très difficiles. Le patient doit être considéré comme responsable de ses choix et de ses comportements, tout en se sentant toujours soutenu par son thérapeute. Un plan de gestion des risques d'automutilation et de suicide doit être développé pour chaque cas, avec le patient et tous les membres de la famille concernés.

Développer des capacités de régulation émotionnelle. Les patients TPB sont sujets à des états émotionnels puissants qu'ils se sentent incapables de gérer, ou qu'ils amplifient à travers des réactions émotionnelles secondaires (ils se mettent par exemple en colère parce qu'ils se sentent tristes). D’un côté, ils semblent tolérer un large éventail d'émotions et montrent pourtant, dans le même temps, une tolérance extrêmement limitée à certaines autres. Ils ont besoin d'apprendre à accepter toutes leurs réactions émotionnelles et à les placer dans leurs contextes situationnels, pour pouvoir comprendre ce qui les déclenche (entre autres des événements passés ou présents). Des notions psychoéducatives concernant les émotions et leurs fonctions sont indispensables pour leur permettre de comprendre que le fait de se sentir blessé ou en colère ne signifie pas qu'ils sont " dégoûtants », « anormaux », ou qu'ils « ont tort ».

Dans les premiers stades de la thérapie, il est essentiel d'encourager l'auto-observation, car les patients TPB ont tendance à se laisser submerger par leurs émotions sans réfléchir à ce qu'elles signifient. Le développement d'un point de vue métacognitif (Fonagy, Gergely, Jurist $\&$ Target, 2002) est une acquisition régulatrice essentielle pour les patients TPB, qui peuvent ainsi devenir conscients de leurs réactions. Ce point de vue « méta » est également essentiel pour développer la capacité d'attention double (envers le passé et le présent) nécessaire au retraitement EMDR efficace des souvenirs dans les phases $4-7$. Une patiente TPB s'était ainsi mise en colère contre elle-même pour s'être ouverte à quelqu'un qui, par la suite, l'avait trahie. Elle rapportait que son émotion première avait été la tristesse, mais qu'elle avait tout de suite commencé à s'adresser des injures pour avoir été faible et trop émotive. Le thérapeute lui demanda de noter si quelque chose changeait lorsqu'elle observait sa tristesse sans la juger, dans une acceptation totale, puis il fit une séquence de SBL. Elle indiqua alors qu'elle pouvait ressentir de la compassion pour elle-même. Après une autre séquence de SBL, sa compassion pour elle-même grandit, et elle commença à réfléchir aux signaux qui auraient pu lui indiquer qu'il ne fallait pas se fier à cette relation. Elle ajouta alors qu'elle pouvait tirer des enseignements de ce qui lui était arrivé.
Quand les patients, par crainte de la réaction négative des autres, évoquent une peur excessive de poser des limites ou de s'occuper d'eux-mêmes, on peut identifier les origines de ces peurs à travers le recueil de l'histoire personnelle du patient ou l'utilisation d'un pont d'affect, et les retraiter alors. Des activités distrayantes peuvent être plus utiles que du travail de confinement, car ces patients n'ont généralement que trop tendance à se contenir, jusqu'au moment où ils explosent. Les stratégies régulatrices tournées vers l'extérieur (les appels téléphoniques désespérés vers les amis ou la famille, la recherche d'un amour " authentique ", etc.) devront graduellement se transformer en un apprentissage de compétences d'autorégulation (à travers un dialogue intérieur soutenant, un changement du centrage attentionnel, etc.) Les solutions magiques doivent être abandonnées au profit de stratégies réalistes. Des procédures favorisant le soin à soi-même peuvent aider ces patients à comprendre leurs états émotionnels, à les accepter, à se considérer avec compassion, sans jugement, et à établir des frontières adaptées dans leurs relations. Ces interventions de régulation émotionnelle ne doivent pas être considérées comme une simple phase de préparation avant le retraitement : les deux peuvent être combinés de façon dynamique, en fonction des particularités de chaque patient. Dans certains cas, la meilleure intervention d'autorégulation consiste à retraiter les expériences traumatiques centrales : l'impression d'être invisible, de n'être l'objet de l'attention de personne ou de n'avoir personne vers qui se tourner pour recevoir soutien et protection.

Le travail centré sur les abus de substances. Les addictions aux drogues et à l'alcool accompagnent fréquemment les TPB (Paris \& Z weig-Frank, 2001 ; Trull, Sher, Minks-Brown, Durbin \& Burr, 2000 ; Zanarini, Frankenburg, Hennen, Reich \& Silk, 2004 ; Zimmerman \& Mattia, 1999) ; elles doivent faire l'objet d'une exploration spécifique. La toxicomanie est souvent liée aux capacités limitées d'autorégulation émotionnelle des individus TPB, et c'est une composante fréquente de la myriade d'interactions complexes qui rendent si difficile le traitement de la pathologie borderline et exigent des approches particulières. Il peut être indiqué, au cours des premières phases du travail, d'adresser ces patients à un traitement de groupe de la toxicomanie comme «Seeking Safety » aux USA (Najavits, 2002 ; Najavits, Weiss, Shaw \& Muenz, 1998), parallèlement à la construction de la relation thérapeutique et d'un plan de traitement favorisant l'abstinence. Un traitement EMDR individuel centré sur le développement de capacités d'adaptation (Korn \& Leeds, 2002) 
et sur le traitement des déclencheurs de rechutes (Hase, 2010 ; Hase, Schallmayer \& Sack, 2008 ; Hofmann, 2004, 2010 ; Popky, 2005) doit parfois être intégré également au plan de traitement.

Il faut parfois adresser dès le début du travail certains patients à des institutions psychiatriques, en traitement hospitalier ou en suivi ambulatoire, pour les aider à se sevrer ou à dépasser le risque de comportements à haut risque liés à leur addiction (par exemple, un coma éthylique ou une overdose). Dans d'autres cas, ce type de traitement aura pu se montrer inefficace à plusieurs reprises, et il faut utiliser le traitement EMDR sur les déclencheurs de rechute actuels (Hase, 2010 ; Hase et coll., 2008 ; Hofmann, 2004, 2010 ; Popky, 2005) ou même sur certains souvenirs traumatiques précoces en rapport avec l'abus de substances. Citons l'exemple d'un homme de 43 ans, alcoolique compulsif, qui présentait des comportements très agressifs lorsqu'il était sous l'emprise de l'alcool. Le risque qu'il blesse quelqu'un étant élevé, son thérapeute l'adressa à un programme résidentiel de traitement de l'alcoolisme. Après un mois d'hospitalisation, il fut renvoyé en raison de fréquents problèmes relationnels avec d'autres patients. Son thérapeute décida alors de tenter le retraitement EMDR d'un souvenir relié à un sentiment d'impuissance et de culpabilité qui précédait ses épisodes d'alcoolisme les plus graves. Le retraitement de ce souvenir mit fin aux comportements problématiques et permit au patient de poursuivre sa thérapie en ambulatoire. Dans certains cas comme celui-ci, le travail de stabilisation, avec des tâches appartenant à la phase de préparation - comme le développement de compétences et l'autorégulation - ne répond pas forcément au besoin du patient d'aborder un ou plusieurs souvenirs qui sous-tendent des comportements perturbés ou des rechutes récurrentes.

Travailler sur les défenses. Au sens le plus général, les défenses psychologiques représentent une gamme de comportements adaptatifs internes et externes, servant à éviter des sentiments et des pulsions conflictuels (McCullough, 2003). Les défenses peuvent être comprises comme des protections, comme des phobies d'affect (McCullough, 2012) ou comme des phobies intéroceptives d'actions mentales dérivées du trauma (Van der Hart et coll., 2006). Il ne faut ni les confronter, ni les ignorer, mais plutôt les aborder progressivement et à un rythme tolérable pour le patient (Gonzalez \& Mosquera, 2012 ; McCullough, 1997). La psychoéducation est fondamentale à cet égard. McCullough (2012) décrit cette étape comme la reconnaissance des défenses.
Les défenses peuvent souvent devenir des alliés de la thérapie lorsque le patient et le thérapeute comprennent leur fonction sous-jacente. Quand le patient est capable d'identifier une défense dont le thérapeute pense qu'elle bloque le processus thérapeutique, il est parfois possible de la retraiter à l'aide d'interventions EMDR spécifiques (Gonzalez \& Mosquera, 2012 ; Knipe, 1999, 2003, 2005, 2009, 2010a, 2010 b, 2010c ; Leeds, 2009, p. 164 ; Leeds, 2012 ; Shapiro, 2001, p. 192-197). C'est le cas d'un patient qui présentait de fréquentes attitudes défensives en thérapie : banalisation, tendance à s'engager dans des conversations superficielles et dans des évitements, annulation des rendez-vous ou retards. Le thérapeute identifia ses tendances à l'évitement comme un problème central et les sélectionna en tant que déclencheurs actuels, ce qui permit de les retraiter. A la suite de cela, l'engagement du patient envers sa thérapie et son respect du cadre thérapeutique s'améliorèrent.

Procédures spécifiques pour la différenciation de soi. Un point important qui doit être abordé en thérapie est la différenciation de soi. Harter (1999) passe en revue différentes perspectives sur la représentation de soi et propose une perspective développementale qui explique comment les premières interactions avec les personnes qui apportent soin et attention au bébé (interactions souvent perturbées chez les patients TPB) façonnent les représentations adultes du soi. De nombreuses personnes souffrant de TPB présentent un manque de différenciation de soi, dans laquelle la perception de soi contient un mélange d'émotions provenant de soi et d'autres personnes. C'est-à-dire que, dans le soi indifférencié des patients TPB, il y a une confusion entre les perceptions internes et externes des émotions. Kernberg (1993) décrit les interventions visant à aborder cette question comme la première étape du traitement des personnalités borderline. Bateman et Fonagy (2004) appellent cette question centrale «l'équivalence psychique ». Plusieurs procédures EMDR particulières servant à travailler la différenciation ont été proposées (Litt, 2007).

Travailler sur les parties dissociatives de la personnalité. On trouve généralement quelque degré de dissociation structurelle (Van der Hart et coll., 2006), même chez des patients TPB ayant des symptômes dissociatifs moins graves. Les patients perçoivent ou décrivent ces parties dissociatives de la personnalité comme « des aspects différents » ou « des réactions qui changent ». Il est utile d'explorer la présence des parties dissociatives de la personnalité à travers des représentations concrètes (dessins, petits personnages, etc.) : cela permet au clinicien de développer et d'affiner sa formulation du cas, et 
au patient de développer des capacités de réflexion et un point de vue métacognitif (Van der Hart, Groenendijk, Gonzalez, Mosquera \& Salomon, 2013, p. 8-9). Ce partage de compréhension de ces différents aspects de la personnalité est essentiel à la réussite du retraitement EMDR des traumas (Gonzalez \& Mosquera, 2012). Dans les cas où la dissociation structurelle n'est pas au premier plan, il n'est pas nécessaire de travailler longtemps sur les parties dissociatives, mais une exploration spécifique de ces aspects au cours de la phase de préparation aide souvent à prévenir ou à résoudre des difficultés dans les phases 3-7.

\section{Retraitement des traumas : phases 3-7}

Avec la plupart des patients TPB qui présentent des capacités d’adaptation limitées et une stabilité émotionnelle minime, il n'est généralement pas souhaitable de commencer le retraitement en abordant leurs pires souvenirs ni aucun de leurs souvenirs traumatiques ou difficiles. Dans beaucoup de ces cas peu stables, on peut néanmoins commencer à utiliser les procédures EMDR standard sur les déclencheurs du présent, comme les réactions émotionnelles face à un partenaire, un collègue de travail ou un supérieur, et ce relativement tôt dans la thérapie (Hofmann, 2010 ; Leeds \& Mosquera, 2012 ; Mosquera, 2012b). Avec des patients TPB plus stables, il est parfois possible de commencer le retraitement d'anciens souvenirs, mais ce n'est pas la règle.

Le rythme du processus thérapeutique. Au début du retraitement, la prise de décision concernant le choix et le séquençage des cibles peut être influencée par de nombreux facteurs, chez le clinicien comme chez le patient (Leeds \& Mosquera, 2012). Certains cliniciens (sous l'influence d'un contre-transfert idéalisé de sauvetage du patient) peuvent avoir tendance à passer trop vite au dévoilement du matériel traumatique sans comprendre préalablement la capacité du patient à gérer ses problèmes quotidiens ou sa difficulté à se confronter au matériel traumatique. Par ailleurs, il arrive au contraire que le patient ou le thérapeute tendent à éviter ou à renvoyer à plus tard le retraitement des traumatismes, à cause d'une phobie de l'affect ou d'une phobie des contenus traumatiques (McCullough, 1997 ; van der Hart et coll., 2006).

Déterminer (tester) quand le patient est prêt pour le traitement.

Tester les étapes procédurales EMDR standard. On peut tenter une utilisation prudente du protocole ESPT complet de l'EMDR (Leeds, 2009 ; Shapiro, 2001) lorsque le patient montre des signes de stabilité (comme décrit dans la section suivante). Cette première tentative peut être présentée comme un test visant à déterminer si le moment est bien choisi pour commencer à aborder directement les souvenirs traumatiques ou négatifs de la personne. Si la première cible est bien choisie, si le patient comprend l'objectif de la procédure et qu'il a des attentes réalistes, les résultats de ce test peuvent guider le clinicien dans la décision de passer au retraitement d'autres cibles.

Tester les étapes procédurales EMDR modifiées. Ce test d'essai peut être fait même chez des patients au fonctionnement bas, en apportant de légères modifications aux étapes procédurales EMDR classiques. Le test peut consister en un retraitement bref et inachevé d'un élément négatif, comme une situation inconfortable du quotidien ou un souvenir récent faiblement perturbant (niveau bas sur l'échelle SUD). Ici, il est important de retourner à la cible après quelques séquences seulement de SBL (ce qui limite les associations) et de stopper la procédure dès que la perturbation diminue, même un peu, ou qu'une prise de conscience apparait. Ce court test ne doit prendre que quelques minutes. Il peut être présenté au patient comme une expérience permettant de tester les effets des SBL sur les expériences perturbatrices négatives et de décider, en concertation avec lui, si les procédures SBL peuvent être utiles en début de thérapie ou si elles doivent être remises à plus tard.

Le thérapeute doit expliquer à son patient que, même s'il s'avère que la thérapie EMDR avec SBL peut être utile dans son cas, le moment propice pour l'utiliser est d'une grande importance. Il doit décrire "l'expérience " comme une situation ayant de nombreux résultats possibles: les sensations positives et négatives sont susceptibles de s'accentuer, de diminuer ou de rester les mêmes, et elles peuvent changer ou non de localisation physique. Les SBL peuvent débloquer des situations dans le cerveau du patient, et on peut le constater de différentes manières : le patient peut avoir une plus grande conscience de ses émotions, observer une accentuation ou une diminution de leur intensité, des souvenirs différents peuvent faire surface . . . et tout cela est bien. Toutes ces réponses possibles donneront des informations sur la façon dont le cerveau du patient traite l'information.

Grâce aux résultats de ce test, le patient et le thérapeute sauront si les procédures du retraitement EMDR peuvent être introduites dès le début du traitement ou si une phase de préparation plus longue est nécessaire avant de recommencer le test, quelques mois plus tard. Le thérapeute doit insister pour que le patient ne force rien ; cela montrera combien il respecte son opinion dans sa prise de décision. Même si 
l'expérience montre que le retraitement EMDR peut être perturbant pour ce patient particulier, la relation thérapeutique peut sortir renforcée du fait que le patient aura appris que la thérapie est basée sur la collaboration, et qu'il en est l'acteur principal.

Sélectionner les cibles. Avec les patients TPB, pour différentes raisons, la sélection des cibles peut être difficile. D'abord, il peut y avoir un grand nombre de cibles pertinentes, avec beaucoup d'expériences négatives et beaucoup d'expériences traumatiques, uniques ou répétées (Leeds \& Mosquera, 2012 ; Mosquera, 2012b). Par ailleurs, dans les cas de trauma complexe, une amnésie ou des souvenirs fragmentés peuvent gêner l'identification de cibles majeures du passé. Et quand on a des souvenirs clairement identifiés, des mécanismes de défense et d'évitement peuvent empêcher qu'on les aborde directement. Lorsque cela se produit, un travail de préparation, de psychoéducation et de stabilisation plus long est parfois nécessaire. Il n'est pas rare que l'évitement phobique et défensif entourant certains souvenirs (Van der Hart et coll., 2013) doive être choisi comme cible initiale pour le retraitement, jusqu'à ce qu'on ait résolu un nombre suffisant de peurs spécifiques et développé une bonne tolérance aux affects.

Déterminer si le patient est prêt. En accord avec le modèle orienté par phase, dont la validité est reconnue (Courtois et coll., 2009 ; Van der Hart et coll., 1989), Hofmann (2010) propose que les patients ayant un ESPT-C très instable doivent répondre aux quatre critères de stabilité suivants avant qu'on puisse procéder à l'identification et au retraitement des souvenirs traumatiques principaux : capacité à prendre correctement soin de soi dans le fonctionnement quotidien, ressources de confinement et d'auto-apaisement, connexion avec ses sensations corporelles, et capacité à verbaliser les souvenirs traumatiques sans se dysréguler. Hofmann suggère que les patients particulièrement instables qui ne répondent pas à ces critères se voient proposer le DIR (Korn \& Leeds, 2002), l'installation permanente de sécurité et d'orientation vers le présent (CIPOS ; Knipe, 2010d) et le retraitement de cibles de la vie quotidienne (déclencheurs du présent), jusqu'à stabilisation.

Malheureusement, les cliniciens qui choisissent d'attendre que ces quatre conditions soient systématiquement réunies avant de commencer le retraitement traumatique EMDR risquent d'attendre des années, voire de ne jamais parvenir à cette "stabilité nécessaire ». Pour de nombreux patients TPB, cette façon " prudente » d'utiliser l'EMDR peut retarder indûment, ou même empêcher, le bénéfice de l'essentiel de la thérapie EMDR (Gonzalez \& Mosquera, 2012).

Dans certains cas où les patients TPB ne possèdent pas de bonnes capacités d'autorégulation et ont des difficultés à prendre soin d'eux-mêmes, le retraitement peut être cependant bien toléré et efficace. La capacité de régulation de ces patients peut être améliorée après un retraitement limité, alors que des interventions moins « intenses », qui diffèrent ou évitent la confrontation au souvenir traumatique — tels que le DIR ou la TCD - peuvent ne pas être aussi efficaces avec eux. Dans certains cas, le patient n'a pas d'autres options ni d'autres ressources à sa disposition. Lorsqu'il en est ainsi, le fait d'identifier les expériences de vie traumatiques ou négatives liées de façon évidente aux comportements les plus risqués et aux problèmes qui affaiblissent la personne, et de les retraiter rapidement au cours de la thérapie peut être l'intervention la plus stabilisatrice. Ainsi, avec certains patients, il est préférable " d'aller lentement » et de suivre une approche prudente ; dans d'autres cas, une approche plus directe est nécessaire. Les informations obtenues grâce aux «tests thérapeutiques » sont nécessaires pour concevoir un plan de travail général.

Dans la mesure où l'organisation individuelle des patients répondant aux critères du TPB, ainsi que leur réceptivité à nos interventions, peuvent être très diverses, il faut proposer chaque intervention comme une expérience et considérer les réactions des patients, en séance et en dehors des séances, comme des informations essentielles pour nos prises de décision cliniques. À mesure que nous voyons les patients TPB tolérer nos interventions et se stabiliser, nous pouvons passer au retraitement des cibles les plus pertinentes, qui sont parfois de plus en plus perturbantes. Nous allons décrire quelques propositions pour structurer le ciblage et le retraitement dans le reste de cet article.

Différentes voies d'accès dans le plan de traitement $\boldsymbol{d} \boldsymbol{u}$ TPB. Les patients TPB présentent des situations différentes, qui influencent le cours que prendra le processus thérapeutique. Avec l'approche progressive, nous pouvons commencer par les tâches les plus tolérables pour le patient et passer à des tâches plus difficiles qui demandent l'utilisation de l'EMDR et des SBL. Généralement, dans le travail avec des patients TPB (excepté avec le sous-type dissociatif), on peut envisager cinq options de base pour les interventions EMDR, de la plus accessible et tolérable à la plus difficile à gérer et à aborder.

1. Donner au patient des notions psychoéducatives et faire avec lui le DIR. Cela est approprié lorsque le thérapeute a jugé que le patient a besoin d’une 
préparation spécifique et n'est pas prêt pour le retraitement d'éléments dysfonctionnels.

2. Explorer, en collaboration avec le patient, les effets des SBL sur des éléments dysfonctionnels limités, simplement dans le but de tester leur efficacité avec ce patient particulier. Ces éléments comprennent des sensations de malaise et des situations de vie problématiques, mais pas trop difficiles.

3. Cibler et retraiter des éléments défensifs dysfonctionnels limités. Cela diffère du retraitement de réseaux entiers de souvenirs spécifiques : il s'agit par exemple de pulsions défensives d'évitement, de pulsions compulsives à visée d'évitement ou de matériel transférentiel.

4. Cibler et retraiter des déclencheurs actuels (en évitant ou différant délibérément la confrontation avec les souvenirs principaux). On sélectionne ces déclencheurs quand le thérapeute juge que le patient n'est pas encore capable de supporter le ciblage de souvenirs anciens, mais qu'il est prêt au retraitement.

5. Cibler et retraiter les souvenirs anciens : le thérapeute les sélectionne lorsqu'il juge son patient capable de retraiter d'anciens souvenirs. Il peut s'agir de souvenirs d'enfance, d'adolescence ou issus de l'âge adulte, choisis pour leur implication dans les aspects les plus difficiles des symptômes actuels du patient. On peut les repérer (a) en faisant le relevé de l'histoire personnelle du patient, (b) en faisant un pont d'affect (Watkins, 1971, 1990), par la technique du floatback (Young, Zangwill \& Behary, 2002, p. 195) ou à partir de déclencheurs actuels, et (c) par des associations spontanées entre des souvenirs récents et un souvenir central plus ancien.

Les caractéristiques des patients qui doivent guider le séquençage des cibles. Il faut garder à l'esprit les caractéristiques spécifiques du patient afin de guider le séquençage des cibles et d'organiser un plan thérapeutique. Voici quelques exemples du processus de prise de décision :

En présence de comportements à haut risque ou de symptômes qui conduisent le patient à décompenser. Afin d'aider le patient à se stabiliser, commencez par des cibles présentes ou passées qui sont clairement associées aux symptômes les plus débilitants du patient et continuez avec les cibles associées à ces symptômes particuliers jusqu'à ce qu'il montre des signes de stabilité, avec une réduction ou une élimination des symptômes associés. L'automutilation, l’agression, les comportements à risque ou les cibles en rapport avec la décompensation doivent être abordés en premier.
Lorsque le patient est prêt à parler de ses souvenirs anciens, même ceux qui suscitent en lui une intense émotion, choisissez et commencez à travailler sur un souvenir précoce que vous et votre patient estimez en rapport avec ses comportements à risque ou avec ses problèmes débilitants. S'il montre qu'il ne peut pas encore supporter de se concentrer sur des cibles plus perturbantes du passé, concentrez-vous sur le retraitement des déclencheurs actuels, en alternant avec de brefs contacts spontanés ou guidés avec les cibles du passé, jusqu'à ce que le patient puisse tolérer un contact plus étendu avec son passé.

En cas de souvenirs, de pensées ou de croyances intrusives. Lorsqu'il y a des souvenirs intrusifs, travaillez sur eux en premier. Quand ces intrusions sont intenses et récurrentes, leur retraitement peut être d'un grand bénéfice pour le patient. Ces souvenirs intrusifs sont souvent si activés que toute tentative de retraiter un autre matériel est vouée à l'échec. Les pensées négatives persistantes ou les croyances limitantes peuvent être des cibles initiales productives, qu'on fera suivre de la recherche d'un souvenir spécifique en utilisant les procédures décrites plus haut.

Lorsqu'il n'y a pas (ou plus) de comportements à risque, ni de souvenirs, de pensées ou de croyances intrusives. Quand les comportements à risques et les intrusions ont été résolus, ou si le patient n'en présente pas, il faut en général choisir les cibles en fonction de sa capacité à supporter le travail sur des souvenirs précoces et perturbants.

Quand les patients rapportent être en contact avec des souvenirs activés (Korn, 2009), il est préférable de sélectionner un souvenir sur lequel ils sont d'accord pour travailler afin de faire baisser la perturbation associée avec celui-ci. C'est toujours un choix plus judicieux qu'un souvenir que le thérapeute croit pertinent dans des situations où (a) il n'y a pas d'accord patient/thérapeute concernant la relation de ce souvenir avec les problèmes du patient, et où (b) le patient n'est pas d'accord pour travailler sur le souvenir que le clinicien a choisi et n'y est pas prêt.

Lorsque les patients ne parviennent pas à se connecter avec des souvenirs anciens perturbants, ou en présence d'une amnésie dissociative, commencez par retraiter les déclencheurs actuels qui leur sont associés.

Lorsqu'on travaille avec des patients TPB, chez qui il est courant de trouver une activation s'élargissant à de nombreuses expériences difficiles associées (Collins \& Loftus, 1975 ; Leeds, 1998) et une tolérance aux affects défaillante, il est généralement important de limiter l'étendue du matériel associé qui émerge pendant le retraitement d'un déclencheur du présent. 
Si ce type de matériel apparaît, le clinicien devra en général répondre en reconnaissant l'importance des cibles plus anciennes, et en ramenant l'attention du patient au déclencheur actuel. Cependant, si le clinicien pense qu'un souvenir que le patient peut tolérer a émergé, il peut envisager de tester la possibilité de poursuivre en travaillant sur ce souvenir, mais $\grave{a}$ condition d'obtenir d'abord la permission du patient et seulement tant que le retraitement centré sur ce souvenir reste tolérable pour ce dernier.

Si des souvenirs intolérables émergent : (a) mettez-les de côté ; (b) si nécessaire, aidez le patient à se restabiliser ; (c) continuez avec le déclencheur actuel (d) à la condition que le patient soit d'accord pour reprendre le retraitement. Le clinicien doit surveiller les signes indirects d'inconfort (nervosité grandissante, agitation, capacités réduites à suivre souplement les mouvements oculaires). Dans la mesure où les patients souffrant d'une dissociation structurelle plus grave peuvent à la fois ne pas être conscients de cet inconfort et n'en montrer aucun signe observable, le clinicien doit être préparé à rechercher les signaux stop implicites auprès d'autres parties du système de la personnalité en utilisant par exemple le scan interne (Gonzalez \& Mosquera, 2012). Gardez à l'esprit que des séquences de travail plus courtes sont préférables quand elles restent dans la fenêtre de tolérance et n'excèdent pas l'énergie mentale disponible du patient. Dans les cas de trauma complexe avec une histoire précoce d'impuissance apprise, il est essentiel et hautement thérapeutique de donner aux patients un rôle actif dans la prise de décision, sans jamais les forcer et en vérifiant régulièrement qu'ils ne soient pas débordés ou épuisés.

Lorsque le patient est prêt à retraiter des souvenirs traumatiques. Quand le patient est (a) suffisamment stable et fort pour faire face à des souvenirs hautement traumatiques, (b) qu'il n'y a pas de défenses particulières ni d'amnésie dissociative et (c) qu'on a procédé à un premier test expérimental avec les SBL, il est possible de cibler ces souvenirs directement, mais les approches progressives décrites ci-dessus sont généralement recommandées en premier lieu.

Adaptations dans la phase d'évaluation. Après avoir sélectionné une cible pour le retraitement, il est important de rester attentif à des précautions supplémentaires pendant la phase 3. Parfois, le travail consistant à sélectionner des cognitions positives et négatives nécessite à la fois une simplification et une aide de la part du thérapeute. S'il est utile de savoir quelle est la cognition négative la plus fortement reliée à la cible, il est essentiel aussi d'empêcher les patients TPB de se perdre complètement sous une avalanche sans fin de croyances négatives sur eux-mêmes. Ces patients ont généralement de nombreuses et puissantes croyances négatives sur eux-mêmes. Ils éprouvent souvent des difficultés lorsqu'il s'agit de ne sélectionner qu'une seule croyance qui serait la plus fortement connectée à une expérience particulière (Leeds \& Mosquera, 2012 ; Mosquera, 2012b). Afin de construire leur capacité à mentaliser (Bateman \& Fonagy, 2004), il est hautement thérapeutique pour les patients TPB de reconnaître que leur notion d'eux-mêmes a émergé d'expériences spécifiques (extérieures à eux). Cet aspect standard de la phase 3 est donc une intervention tout à fait pertinente et potentiellement adaptée qu'il faut garder de la procédure EMDR standard. Cependant, les patients qui ont tendance à être perturbés par cette étape auront besoin d'une aide plus grande de leur thérapeute pour ne pas se trouver submergés ou égarés. Par exemple, ce dernier pourra dire : "Je sais que c'est difficile et que vous avez peut-être beaucoup de phrases négatives en tête, mais essayez de penser à la situation et de choisir la phrase qui lui correspond le mieux. Prenez le temps qu'il vous faut. » Ou encore : "Quand vous vous concentrez sur ce souvenir et sur les émotions qu'il fait venir en vous, est-ce qu'il s'agit plutôt d'une impression de perdre le contrôle, d'une impression d'être en danger immédiat ou d'une impression de ne rien valoir? " En même temps, lors de cette étape, les thérapeutes doivent faire attention à ne pas faire le travail à la place des patients en leur proposant tout de suite, par exemple, une cognition négative particulière. On peut inclure ici des informations psychoéducatives et il faut stimuler la réflexion du patient.

Il n'est pas rare, chez les patients TPB, qu'il soit plus difficile de trouver une cognition positive (CP) qu'une cognition négative. En effet, les patients qui ont manqué d'expériences précoces de partage d'affects positifs et de reconnaissance mutuelle (Leeds, 2006), n'ont souvent pas une tolérance aux affects suffisante, ni une image d'eux-mêmes assez positive pour pouvoir exprimer la moindre phrase d'affirmation de soi. Dans certains cas, un travail particulier sur la tolérance aux affects positifs pourra être utile avant de passer au retraitement traumatique. Dans d'autres cas, la CP initialement proposée par le patient peut être une pensée irréaliste, idéalisée, qui doit être modifiée sur les conseils du thérapeute, à travers une discussion en collaboration avec le patient. En voici quelques exemples simples : "Ce n’est jamais arrivé ", "Je peux tout contrôler " ou " Je suis invincible ". Exemple plus subtil : le patient donne comme CP : "Je suis fort ", alors que sa cognition négative était : 
«Il n'est pas prudent d'exprimer ses besoins ou de demander de l'aide. " Ici, l'idée d'être " fort » est en fait une défense contre la vulnérabilité que représente le fait d'exprimer ses besoins. Dans tous les cas, une CP adaptée doit être trouvée avant de procéder à la phase 4 (désensibilisation), sous peine d'exposer le patient à un retraitement qui aille du négatif au négatif, sans qu'il y ait jamais de connexion avec des réseaux de souvenirs positifs. Si aucune CP forte n'est possible (ex. : "Je suis quelqu'un de bien »), une cognition de transition peut être choisie, telle que : «Je peux apprendre à m'accepter comme je suis ».

\section{Les adaptations dans la phase de désensibilisation.} Construction d'une structure face à l'activation expansive du patient. Avec les patients TPB, la nécessité de maintenir une structure (c'est-à-dire une concentration) pendant la désensibilisation, en phase 4 , constitue une difficulté importante. Ces patients ont en effet tendance à présenter une "activation expansive » (Collins \& Loftus, 1975 ; Leeds, 1998) : un réseau mnésique dysfonctionnel se relie à beaucoup d'autres, et beaucoup plus rarement (voire jamais) à des réseaux de souvenirs adaptés. Par conséquent, dans ces cas de troubles du stress extrême, l'instruction EMDR standard consistant à commencer le retraitement par ces mots : «Laissez venir tout ce qui vous vient » n'est pas nécessairement une bonne idée. Ces patients vivent dans le chaos et peuvent avoir plutôt une " liste de 100 souvenirs les plus perturbants » qu'une liste de 10 . Chez de nombreux patients TPB, si le clinicien suit simplement le processus d'associations spontanées, il arrive facilement que trop de matériel s'active sans que cela produise un retraitement efficace. Des variations par rapport aux lignes directrices du retraitement standard ont été proposées par plusieurs auteurs (Gelinas, 2003 ; Korn, 2009 ; Leeds, 2009 ; Paulsen, 1995); elles ressemblent un peu à l'EMDR originelle (Shapiro, 1989), où l'on revenait à la cible après chaque séquence de SBL, en utilisant des chaînes associatives courtes : après seulement deux ou trois séquences de SBL, ou dès qu'un ou deux nouveaux souvenirs (négatifs) émergent par association spontanée, on retourne à la cible et on demande : "Qu'est-ce qui est différent? 》 (afin d'éviter la réponse automatique : «Pareil»).

Utilisation répétée des tissages afin d'activer des informations adaptées. L'activation délibérée d'informations adaptées, également connue sous le nom de tissages (Leeds, 2009) ou de tissages cognitifs (Shapiro, 2001), peut être faite de façon répétitive avant les séquences suivantes de SBL lorsqu'on a besoin d'aborder un retraitement inefficace. Le thérapeute peut ainsi introduire les éléments d'un changement positif dans les croyances, par exemple : «Qu'est-ce qui pourrait vous aider à avoir une meilleure opinion de vous-même ? " Afin d'aider le patient à progresser vers la réflexion, la mentalisation et la réalisation, en revenant à la cible, le thérapeute peut poser une des questions suivantes : "Qu'est-ce que vous comprenez de cette expérience maintenant ? ", "Que pensez-vous de cette expérience maintenant ? ", " De quoi êtes-vous conscient maintenant, dont vous n'aviez pas conscience alors ? " Lors du retraitement, avec des patients souffrant d'un ESPT suite à une expérience unique ou un petit nombre d'expériences traumatiques, le lien entre information adaptée et réalisation se produit souvent spontanément. Avec des patients souffrant de troubles du stress extrême, on peut favoriser activement cette connexion qui risque ne pas se produire autrement, à cause du faible nombre d'expériences adaptées qu'ils ont connues dans leur enfance.

Exploration active des émotions secondaires qui peuvent perturber le retraitement. Une autre façon de favoriser un retraitement efficace est d'aider le patient à faire face aux émotions secondaires qui émergent fréquemment chez les personnes atteintes de TPB. Ces émotions secondaires sont liées à des jugements ou des croyances négatives qui sont activés à cause d'un état émotionnel primaire (voir Leeds, 2009, p. 164 ; Shapiro, 2001, p. 192-193). Par exemple, lorsque le patient ressent une rage en lui, il peut se dire : "Je suis mauvais " (tout comme l'était son agresseur furieux). La croyance « je suis mauvais » est associée à des sentiments secondaires de honte. Cette émotion secondaire peut perturber le retraitement. Ces perturbations peuvent être indiquées par des déclarations de type : «Rien ne me vient », par l'apparition soudaine de symptômes somatiques, par une logorrhée, par une augmentation de l'anxiété ou par des montées perturbantes du niveau d'activation émotionnelle du patient.

Après chaque séquence de $\mathrm{SBL}$, après avoir posé la question habituelle (" Que remarquez-vous maintenant ? ") et entendu la réponse du patient, chaque fois que le thérapeute observe une baisse du flux associatif, il peut être utile de poser une nouvelle question afin d'identifier d'éventuels conflits internes tels qu'une phobie des affects ou une phobie dissociative : «Est-ce que quelque chose d'autre se passe en vous maintenant ? " Cela peut aider à repérer des informations pertinentes et l'éventuelle nécessité d'une intervention supplémentaire. Cela peut également aider le patient à élargir sa perspective et à prendre une position métacognitive. Selon chaque situation, plusieurs interventions possibles peuvent aider le patient à recouvrer la " posture de pleine conscience » nécessaire à un retraitement 
efficace. Citons en exemple des déclarations comme : "Ne critiquez pas de ce qui vient », «Votre propre rage n'était pas la cause des violences psychologiques que votre ... (l'agresseur) vous a fait subir ", "Vous avez vraiment besoin de cette émotion aujourd'hui ? " (les patients pensent parfois avoir besoin de se cramponner à une ancienne émotion), "Autorisez-vous à éprouver toutes vos émotions ", ou "Laissez ça s'exprimer, pas de problème. "

La qualité de l'alliance thérapeutique. Pendant la phase de désensibilisation, le clinicien doit rester attentif aux moments de désaccordage et aux difficultés relationnelles avec son patient. Nous l'avons évoqué plus haut : pour les patients TPB, le facteur essentiel pour un retraitement bien structuré est la présence d'un thérapeute bien ancré, calme, ferme, soutenant le patient et ayant une relation thérapeutique suffisamment forte avec lui. À partir de ce point de départ, diverses interventions techniques peuvent être très utiles, mais elles peuvent aussi être complètement inefficaces sans une alliance thérapeutique adéquate.

Clôturer la séance. Les étapes procédurales et les principes standard de l'EMDR, pour les individus ayant un ESPT sans TPB ni DESNOS, tendent à arriver à clôturer chaque séance. Toutefois, si on propose le retraitement à des personnes souffrant d'un TPB, il vaut mieux privilégier une quantité modeste d'un travail de qualité et finir sur une séance incomplète, que de s'efforcer à tout prix de terminer le retraitement d'une cible spécifique (Leeds \& Mosquera, 2012). En effet, en travaillant avec ce type de patients, il est souvent prudent (voire nécessaire) de mettre fin aux séances alors qu'elles sont techniquement incomplètes. Lorsqu'une séance est incomplète, le thérapeute peut envisager une série d'étapes conduisant à sa clôture : questions au patient pour évaluer son niveau d'activation émotionnelle et somatique ; interventions visant l'auto-apaisement, l'ancrage et la présentification ; examen de la séance en mettant l'accent sur la "mentalisation "; brève installation de phrases métacognitives reflétant les éventuelles avancées thérapeutiques; et suggestions de travail personnel à faire ou répétitions autour de façon de s'occuper de soi-même.

Une installation partielle pour soutenir l'observation $d u$ changement et la mentalisation. Après s'être assuré que le patient se sent bien orienté, qu'il a obtenu un certain contrôle émotionnel et qu'il est capable de réfléchir sur la séance, le thérapeute peut poser des questions sur ce qu'il a le sentiment d'avoir acquis, sur ses prises de conscience ou sur ce qu'il a compris de cette séance. Ensuite, le thérapeute peut envisager
" d'installer " rapidement une réussite ou une prise de conscience qui se seront produites au cours de la séance, ou une ressource qui pourrait aider le patient à faire face au souvenir ; ce genre d'installation comprend en général seulement deux ou trois séquences de SBL, et on n'en attend pas une VoC (validité de la cognition) à 7. La déclaration verbale exprimant le progrès ou la prise de conscience doit, bien sûr, être réaliste. Par exemple, si le patient demande l'installation de sa phrase : "Ce n'est jamais arrivé ", ce n’est pas acceptable. Mais le thérapeute peut proposer une déclaration plus adaptée du type : «Un jour, j’aurai dépassé cela . . . un jour, cela ne me fera plus souffrir ", ou toute autre possibilité adaptée en laquelle le patient peut effectivement croire à ce moment.

\section{Réévaluation}

La plupart des cibles nécessiteront plusieurs séances pour être complètement retraitées. Pendant la phase de réévaluation qui ouvre les séances suivantes, il est essentiel de vérifier son travail en posant des questions sur ce qu'a vécu le patient après la séance, ainsi que sur la cible spécifique abordée lors de la séance précédente. Lorsqu'on décide de ce qu'on va faire, il est important de ne pas perdre de vue son objectif et d'éviter « le saut d'une cible à l'autre " (Mosquera, 2012b). À chaque séance, le patient peut en effet apporter différents points à travailler, mais il est important de trouver un équilibre entre ce qui soucie actuellement le patient et la poursuite du travail sur les cibles qu'on a dûment sélectionnées auparavant (Leeds \& Mosquera, 2012). Il est important d'avoir à l'esprit un plan thérapeutique clair, et cependant souple, dans la mesure où ces patients fonctionnent souvent de façon très chaotique.

\section{Conclusions}

L'EMDR est une thérapie prometteuse pour les patients TPB, mais son application nécessite plusieurs adaptations des procédures EMDR standard, qui ont été développées à l'origine pour des cas plus simples d'ESPT. C'est par les techniques visant la dissociation structurelle qu'on abordera le mieux le sous-groupe des patients TPB qui en présentent les caractéristiques les plus marquées (Gonzalez \& Mosquera, 2012). L'expérience clinique donne à penser que de nombreux patients TPB n'ont besoin que d'un nombre limité d'interventions de stabilisation, ainsi que de modifications mineures des procédures EMDR standard, pour aborder et retraiter en toute sécurité le matériel traumatique, comme on l'a décrit dans le présent article. Une question essentielle, dans le développement et l'organisation de plans de traitement EMDR sûrs 
et efficaces pour les patients TPB, est le développement des compétences conceptuelles, perceptives, procédurales et intersubjectives dont les cliniciens ont besoin pour adapter le travail au rythme du patient dans chacune des huit phases de l'EMDR. Le développement de ces compétences requiert une formation spécialisée avancée et des supervisions personnelles. Des cas publiés précédemment (Brown \& Shapiro, 2006 ; Wesselmann \& Potter, 2009) ainsi qu’une série de cas à paraitre (Mosquera, Gonzalez \& Leeds, 2014) documentent des résultats positifs dans l'application de l'EMDR telle qu'elle est décrite dans cet article. Nous estimons que d'autres recherches sont nécessaires pour examiner les principes énumérés dans ce résumé. Par exemple, on pourrait, sur la base de ces principes, assigner de façon aléatoire des individus répondant aux critères du TPB à deux groupes, avec un même nombre de séances de TCD ou de thérapie EMDR menée sur la base de ces principes.

\section{Bibliographie}

Adler-Tapia, R., \& Settle, C. (2009). Evidence of the efficacy of EMDR with children and adolescents in individual psychotherapy: A review of the research published in peer-reviewed journals. Journal of EMDR Practice and Research, 3(4), 232-247. http://dx.doi .org/10.1891/1933-3196.3.4.232

Agrawal, H. R., Gunderson, J., Holmes, B. M., \& LyonsRuth, K. (2004). Attachment studies with borderline patients: A review. Harvard Review of Psychiatry, 12(2), 94-104.

Bakermans-Kranenburg, M. J., Van Ijzendoorn, M. H., Bokhorst, C. L., \& Schuengel, C. (2004). The importance of shared environment in infant-father attachment: A behavioral genetic study of the attachment q-sort. Journal of Family Psychology, 18(3), 545-549.

Ball, J. S., \& Links, P. S. (2009). Borderline personality disorder and childhood trauma: Evidence for a causal relationship. Current Psychiatry Reports, 11(1), 63-68.

Barone, L., Fossati, A., \& Guiducci, V. (2011). Attachment mental states and inferred pathways of development in borderline personality disorder: A study using the adult attachment interview. Attachment \& Human Development, 13(5), 451-469. http://dx.doi.org/10.1080/14616 734.2011 .602245

Bateman, A., \& Fonagy, P. (2004). Psychotherapy for borderline personality disorder: Mentalization-based treatment. Oxford, United Kingdom: Oxford University Press.

Battle, C. L., Shea, M. T., Johnson, D. M., Yen, S., Zlotnick, C., Zanarini, M. C., . . . Morey, L. C. (2004). Childhood maltreatment associated with adult personality disorders: Findings from the Collaborative Longitudinal Personality Disorders Study. Journal of Personality Disorders, 18, 193-211.
Bisson, J., \& Andrew, M. (2007). Psychological treatment of post-traumatic stress disorder (PTSD). Cochrane Database of Systematic Reviews, 18(3), CD003388. http:// dx.doi.org/10.1002/14651858.CD003388.pub3

Bokhorst, C. L., Bakermans-Kranenburg, M. J., Fearon, R. M. P., Van Ijzendoorn, M. H., Fonagy, P., \& Schuengel, C. (2003). The importance of shared environment in mother-infant attachment security: A behavioral genetic study. Child Development, 74(6), 1769-1782.

Briere, J. (2004). Inventory of Altered Self-Capacities. Lutz, Florida: Psychological Assessment Resources.

Brown, S., \& Shapiro, F. (2006). EMDR in the treatment of borderline personality disorder. Clinical Case Studies, 5(5), 403-420.

Brussoni, M. J., Jang, K. L., Livesley, W. J., \& Macbeth, T. M. (2000). Genetic and environmental influences on adult attachment styles. Personal Relationships, 7, 283-289.

Chu, J. A., \& Dill, D. L. (1991). Dissociation, borderline personality disorder, and childhood trauma. The American Journal of Psychiatry, 148, 812-813.

Classen, C., Pain, C., Field, N., \& Woods, P. (2006). Posttraumatic personality disorder: A reformulation of the complex posttraumatic stress disorder and borderline personality disorder. Psychiatric Clinics of North America, 29, 87-112.

Cohen, P., Crawford, T. N., Johnson, J. G., \& Kasen, S. (2005). The children in the community study of developmental course of personality disorder. Journal of Personality Disorders, 19, 466-486.

Collins, A. M., \& Loftus, E. F. (1975). A spreading activation theory of semantic processing. Psychological Bulletin, 82, 407-428.

Courtois, C. A., Ford, J. D., \& Cloitre, M. (2009). Best practices in psychotherapy for adults. In C. A. Courtois \& J. D. Ford (Eds.), Treating complex traumatic stress disorders: An evidence-based guide (p. 82-103). New York, NY: Guilford Press.

Crawford, T. N., Livesley, W. J., Jang, K. L., Shaver, P. R., Cohen, P., \& Ganiban, J. (2007). Insecure attachment and personality disorder: A twin study of adults. European Journal of Personality, 21, 191-208.

Dalenberg, C. J. (2000). Countertransference and the treatment of trauma. Washington, DC: American Psychological Association.

Driessen, M., Beblo, T., Reddemann, L., Rau, H., Lange, W., Silva, A., . . Ratzka, S. (2002). Is the borderline personality disorder a complex post-traumatic stress disorder? The state of research. Nervenarzt, 73(9), 820-829.

Dworkin, M. (2005). EMDR and the relational imperative: The therapeutic relationship in EMDR treatment. New York, NY: Routledge.

Field, A., \& Cottrell, D. (2011). Eye movement desensitization and reprocessing as a therapeutic intervention for traumatized children and adolescents: A systematic review of the evidence for family therapists. Journal of Family Therapy, 33(4), 374-388. http: / / dx.doi .org/10.1111/j.1467-6427.2011.00548.x 
Foa, E. B., Keane, T. M., Friedman, M. J., \& Cohen, J. A. (2009). Effective treatments for PTSD: Practice guidelines from the International Society for Traumatic Stress Studies. New York, NY : Guilford Press.

Fonagy, P., Gergely, G., Jurist, E. L., \& Target, M. (2002). Affect regulation, mentalization, and the development of the self. New York, NY: Other Press.

Galletly, C. (1997). Borderline-dissociation comorbidity. American Journal of Psychiatry, 154, 1629.

Gelinas, D. J. (2003). Integrating EMDR into phase-oriented treatment for trauma. Journal of Trauma and Dissociation, 4(3), 91-135.

Gianoli, M. O., Jane, J. S., O’Brien, E., \& Ralevski, E. (2012). Treatment for comorbid borderline personality disorder and alcohol use disorders: A review of the evidence and future recommendations. Experimental and Clinical Psychopharmacology, 20(4), 333. http:/ / dx.doi.org/10. 1037 / a0027999

Goldsmith, H. H., \& Harman, C. (1994). Temperament and attachment: Individuals and relationships. Current Directions in Psychological Science, 3, 53-57.

Golier, J., Yehuda, R., Bierer, L., Mitropoulou, V., New, A., Schmeidler, J., . . . Siever, J. (2003). The relationship of borderline personality disorder to posttraumatic stress disorder and traumatic events. American Journal of Psychiatry, 160, 2018-2024.

Gonzalez, A., \& Mosquera, D. (2012). EMDR and dissociation: The progressive approach. Charleston, SC: Amazon Imprint.

Gonzalez, M., Mosquera, D., \& Leeds, A. (2011). Family experiences in childhood scale. [En ligne] http://www intra-tp.org

Goodman, M., \& Yehuda, R. (2002). The relationship between psychological trauma and borderline personality disorder. Psychiatric Annals, 33, 337-345.

Grover, K. E., Carpenter, L. L., Price, L. H., Gagne, G. G., Mello, A. F., Mello, M. F., \& Tyrka, A. R. (2007). The relationship between childhood abuse and adult personality disorder symptoms. Journal of Personality Disorders, 21(4), 442-447.

Gunderson, J. G., \& Sabo, A. (1993). The phenomenological and conceptual interface between borderline personality disorder and post-traumatic stress disorder. American Journal of Psychiatry, 150, 19-27.

Harter, S. (1999). The construction of the self: A developmental perspective. New York, NY: Guilford Press.

Hase, M. (2010). CravEx: An EMDR approach to treat substance abuse and addiction. In M. Luber (Ed.), Eye movement desensitization and reprocessing (EMDR) scripted protocols: Special populations (p. 467-488). New York, NY: Springer Publishing.

Hase, M., Schallmayer, S., \& Sack, M. (2008). EMDR reprocessing of the addiction memory: Pretreatment, posttreatment, and 1-month follow-up. Journal of EMDR Practice and Research, 2(3), 170-179. http: / / dx.doi .org/10.1891/1933-3196.2.3.170
Herman, J. L. (1992). Complex PTSD: A syndrome in survivors of prolonged and repeated trauma. Journal of Traumatic Stress, 5(3), 377-391.

Hesse, E. (1999). The adult attachment interview: Historical and current perspectives. In J. Cassidy \& P. R. Shaver (Eds.), Handbook of attachment: Theory, research and clinical applications (p. 395-433). New York, NY: Guilford Press.

Hill, A. B. (1965). The environment and disease: Association or causation? Proceedings of the Royal Society of Medicine, 58, 295-300.

Hofmann, A. (2004). EMDR in the treatment of complex PTSD. Paper presented at the EMDR International Association Conference, Montreal, Quebec.

Hofmann, A. (2010). The inverted EMDR standard protocol for unstable complex post-traumatic stress disorder. In M. Luber (Ed.), Eye movement desensitization and reprocessing (EMDR) scripted protocols: Special populations (p. 313-328). New York, NY: Springer Publishing.

Horesh, N., Ratner, S., Laor, N., \& Toren, P. (2008). A comparison of life events in adolescents with major depression, borderline personality disorder and matched controls: A pilot study. Psychopathology, 41(5), 300-306. http: / / dx.doi.org/10.1159/000141925

Johnson, J. G., Cohen, P., Brown, J., Smailes, E. M., \& Bernstein, D. P. (1999). Childhood maltreatment increases risk for personality disorders during early adulthood. Archives of General Psychiatry, 56, 600-606.

Kernberg, O. F. (1993). Severe personality disorders: Psychotherapeutic strategies. New Haven, CT: Yale University Press.

Knipe, J. (1999). Targeting defensive avoidance and dissociated numbing. EMDRIA Newsletter, 4(2), 10, 25.

Knipe, J. (2003). "It was a golden time ...”: Treating narcissistic vulnerability. In P. Manfield (Ed.), EMDR casebook (p. 296-319). New York, NY: Norton.

Knipe, J. (2005). Targeting positive affect to clear the pain of unrequited love, codependence, avoidance, and procrastination. In R. Shapiro (Ed.), EMDR solutions: Pathways to healing (p. 189-213). New York, NY: Norton.

Knipe, J. (2009). "Shame is my safe place": AIP targeting of shame as a psychological defense. In R. Shapiro (Ed.), EMDR solutions II: For depression, eating disorders, performance, and more (p. 49-89). New York, NY: Norton Professional Books.

Knipe, J. (2010a). Dysfunctional positive affect: Codependence or obsession with self-defeating behavior. In M. Luber (Ed.), Eye movement desensitization and reprocessing (EMDR) scripted protocols: Special populations (p. 463-465). New York, NY: Springer Publishing.

Knipe, J. (2010b). Dysfunctional positive affect: Procrastination. In M. Luber (Ed.), Eye movement desensitization and reprocessing (EMDR) scripted protocols: Special populations (p. 453-458). New York, NY: Springer Publishing.

Knipe, J. (2010c). Dysfunctional positive affects: To assist clients with unwanted avoidance defenses. In M. Luber (Ed.), Eye movement desensitization and reprocessing (EMDR) scripted protocols: Special populations (p. 451-452). New York, NY: Springer Publishing. 
Knipe, J. (2010d). The method of constant installation of present orientation and safety [CIPOS]. In M. Luber (Ed.), Eye movement desensitization and reprocessing (EMDR) scripted protocols: Special populations (p. 235-241). New York, NY: Springer Publishing.

Korn, D. L. (2009). EMDR and the treatment of complex PTSD: Areview.Journal of EMDRPracticeandResearch,3(4), 264-278. http: / / dx.doi.org/10.1891/1933-3196.3.4.264

Korn, D. L., \& Leeds, A. M. (2002). Preliminary evidence of efficacy for EMDR resource development and installation in the stabilization phase of treatment of complex posttraumatic stress disorder. Journal of Clinical Psychology, 58(12), 1465-1487. http://dx.doi.org/10.1002/ jclp.10099

Korzekwa, M. I., Dell, P. F., \& Pain, C. (2009). Dissociation and borderline personality disorder: An update for clinicians. Current Psychiatry Reports, 11(1), 82-88.

Laporte, L., \& Guttman, H. (1996). Traumatic childhood experiences as risk factors for borderline and other personality disorders. Journal of Personality Disorders, 10, 247-259.

Leeds, A. M. (1998). Lifting the burden of shame: Using EMDR resource installation to resolve a therapeutic impasse. In P. Manfield (Ed.), Extending EMDR, A case book of innovative applications (p. 256-282). New York, NY: Norton.

Leeds, A. M. (2006, September). Learning to feel good about positive emotions with the positive affect tolerance and integration protocol. Paper presented at the annual meeting of the 11th EMDR International Association, Philadelphia, PA.

Leeds, A. M. (2009). A guide to the standard EMDR protocols for clinicians, supervisors, and consultants. New York, NY: Springer Publishing.

Leeds, A. M. (2012, November). Defense and Affect Restructuring in EMDR. Workshop presented for EMDR Spain, Madrid.

Leeds, A. M., \& Mosquera, D. (2012, October). Borderline Personality Disorder and EMDR. Workshop presented at the 2012 EMDRIA Conference, "Attachment: Healing Developmental Trauma”, Washington, DC.

Lieb, K., Völlm, B., Rücker, G., Timmer, A., \& Stoffers, J. M. (2010). Pharmacotherapy for borderline personality disorder: Cochrane systematic review of randomised trials. The British Journal of Psychiatry, 196(1), 4-12. http: / / dx.doi.org/10.1192/bjp.bp.108.062984

Linehan, M. M. (1993). Skills training manual for treating borderline personality disorder. New York,: Guilford Press.

Litt, B. (2007). The child as identified patient: Integrating contextual therapy and EMDR. In F. Shapiro, F. W. Kaslow, \& L. Maxfield (Eds.), Handbook of EMDR and family therapy processes (p. 306-324). Hoboken, NJ: Wiley.

Mccullough, L. (1997). Changing character: Short-term anxiety-regulating psychotherapy for restructuring defenses, affects, and attachment. New York, NY: Basic Books.

Mccullough, L. (2003). Treating affect phobia: A manual for short-term dynamic psychotherapy. New York, NY: Guilford Press.
Mclean, L. M., \& Gallop, R. (2003). Implications of childhood sexual abuse for adult borderline personality disorder and complex posttraumatic stress disorder. The American Journal of Psychiatry, 160, 369-371.

Mosquera, D. (2004). Diamantes en Bruto II. Programa psicoeducativo para personas con Trastorno Limite de la Personalidad. Madrid, Spain: Ediciones Pléyades.

Mosquera, D. (2012a, April). Installing self-care patterns in BPD. A crucial step for achieving stabilization. Workshop presented at Aula Complutense de la Personalidad, Madrid.

Mosquera, D. (2012 b, March). Treating borderline personality disorder with EMDR. Workshop presented at the National EMDR Congress in Holland, The Netherlands.

Mosquera, D., \& Gonzalez, A. (2011a, June). Personality disorders and EMDR. Workshop presented at the Twelfth EMDR Europe Conference in Vienna, Austria.

Mosquera, D., \& Gonzalez, A. (2011 b). Personality disorders, relational trauma and EMDR. Workshop presented at the National EMDR Congress in Rome, Italy.

Mosquera, D., Gonzalez, A., \& Leeds, A. (2014). EMDR therapy for borderline personality disorder: A case series. Journal of EMDR Practice and Research. Manuscrit soumis à la publication.

Mosquera, D., Gonzalez, A., \& Van Der Hart, O. (2011). Borderline personality disorder, childhood trauma and structural dissociation of the personality. Revista Persona, 11 (Suppl. 1), 1-73.

Najavits, L. (2002). The Guilford substance abuse series: Seeking safety: A treatment manual for PTSD and substance abuse. New York, NY: Guilford Press.

Najavits, L. M., Weiss, R. D., Shaw, S. R., \& Muenz, L. R. (1998). "Seeking Safety": Outcome of a new cognitivebehavioral psychotherapy for women with posttraumatic stress disorder and substance dependence. Journal of Traumatic Stress, 11, 437-456.

O’Connor, T. G., \& Croft, C. M. (2001). A twin study of attachment in preschool children. Child Development, 72, 1501-1511.

Pagura, J., Stein, M. B., Bolton, J. M., Cox, B. J., Grant, B., \& Sareen, J. (2010). Comorbidity of borderline. Journal of Psychiatric Research, 44(16), 1190-1198.

Paris, J., \& Zweig-Frank, H. (1997). Dissociation in patients with borderline personality disorder. The American Journal of Psychiatry, 154, 137-138.

Paris, J., \& Zweig-Frank, H. (2001). A 27-year follow-up of patients with borderline personality disorder. Comprehensive Psychiatry, 42, 482-487.

Paulsen, S. (1995). Eye movement desensitization and reprocessing: Its cautious use in the dissociative disorders. Dissociation, 8(1), 32-44.

Pearlman, L. A., \& Courtois, C. A. (2005). Clinical applications of the attachment framework: Relational treatment of complex trauma. Journal of Traumatic Stress, 18(5), 449-459. 
Pietrzak, R. H., Goldstein, R. B., Southwick, S. M., \& Grant, B. F. (2011). Personality disorders associated with full and partial posttraumatic stress disorder in the U.S. population: Results from Wave 2 of the National Epidemiologic Survey on Alcohol and Related Conditions. Journal of Psychiatric Research, 45(5), 678-686.

Popky, A. J. (2005). DeTUR, an urge reduction protocol for addictions and dysfunctional behaviors. In R. Shapiro (Ed.), EMDR solutions: Pathways to healing (p. 167-188). New York, NY: Norton.

Ross, C. A. (2007). Borderline personality disorder and dissociation. Journal of Trauma \& Dissociation, 8(1), 71-80.

Roth, S., Newman, E., Pelcovitz, D., Van Der Kolk, B., \& Mandel, F. S. (1997). Complex PTSD in victims exposed to sexual and physical abuse: Results from the DSM-IV field trial for posttraumatic stress disorder. Journal of Traumatic Stress, 10, 539-556.

Sabo, A. N. (1997). Etiological Significance Of Associations Between Childhood Trauma And Borderline personality disorder: Conceptual and clinical implications. Journal of Personality Disorders, 11, 50-70.

Shapiro, F. (1989). Eye movement desensitization: A new treatment for post-traumatic stress disorder. Journal of Behavior Therapy and Experimental Psychiatry, 20, 211-217.

Shapiro, F. (2001). Eye movement desensitization and reprocessing, basic principles, protocols and procedures. New York, NY: Guilford Press.

Shapiro, F. (2007). EMDR, adaptive information processing, and case conceptualization. Journal of EMDR Practice and Research, 1(2), 68-87. http://dx.doi.org/10.1891/ 1933-3196.1.2.68

Shapiro, F. (2010, March). Mente humana, psicoterapia y EMDR [Human mind, psychotherapy and EMDR]. Paper presented at the XVII Scientific Symposium "From Neurobiology to Nosology of Mental Disorders," Lilly Foundation, Madrid, Spain.

Siever, L. J., Torgersen, S., Gunderson, J. G., Livesley, W. J., \& Kendler, K. S. (2002). The borderline diagnosis III: Identifying endophenotypes for genetic studies. Biological Psychiatry, 51(12), 964-968.

Timmerman, I. G., \& Emmelkamp, P. M. (2001). The relationship between traumatic experiences, dissociation, and borderline personality pathology among male forensic patients and prisoners. Journal of Personality Disorders, 15(2), 136-149.

Trull, T. J., Sher, K. J., Minks-Brown, C., Durbin, J., \& Burr, R. (2000). Borderline personality disorder and substance use disorders: A review and integration. Clinical Psychology Review, 20, 235-253.

Tyrka, A. R., Wyche, M. C., Kelly, M. M., Price, L. H., \& Carpenter, L. L. (2009). Childhood maltreatment and adult personality disorder symptoms: Influence of maltreatment type. Psychiatry Research, 165(3), 281-287.

Ursano, R. J., Bell, C., Eth, S., Friedman, M., Norwood, A., Pfefferbaum, B., . . . Yager, J. (2004). Practice guideline for the treatment of patients with acute stress disorder and posttraumatic stress disorder. American Journal of Psychiatry, 161(11 Suppl.), 3-31.

Van der Hart, O., Brown, P., \& Van Der Kolk, B. A. (1989). Pierre Janet's treatment of post-traumatic stress. Journal of Traumatic Stress, 2, 379-395.

Van der Hart, O., Groenendijk, M., Gonzalez, A., Mosquera, D., \& Solomon, R. (2013). Dissociation of the personality and EMDR therapy in complex trauma-related disorders: Applications in the stabilization phase. Journal of EMDR Practice and Research, 7(2), 81-94. http: / / dx.doi. org/10.1891/1933-3196.7.2.81

Van der Hart, O., Nijenhuis, E. R. S., \& Steele, K. (2006). The haunted self: Structural dissociation and the treatment of chronic traumatization. New York, NY : Norton. Trad. fr. (2010) : Le Soi Hanté. Paris, Le Seuil.

Van der Kolk, B., Roth, S., Pelcovitz, D., Sunday, S., \& Spinazzola, J. (2005). Disorders of extreme stress: The empirical foundation of a complex adaptation to trauma. Journal of Traumatic Stress, 18(5), 389-399.

Van der Kolk, B. A., Spinazzola, J., Blaustein, M. E., Hopper, J. W., Hopper, E. K. Korn, D. L., \& Simpson, W. B. (2007). A randomized clinical trial of eye movement desensitization and reprocessing (EMDR), fluoxetine, and pill placebo in the treatment of posttraumatic stress disorder: Treatment effects and long-term maintenance. Journal of Clinical Psychiatry, 68(1), 37-46.

Watkins, J. G. (1971). The affect bridge: A hypnoanalytic technique. Journal of Clinical and Experimental Hypnosis, 19(1), 21-27.

Watkins, J. G. (1990). Watkins' affect or somatic bridge. In D. C. Hammond (Ed.), Handbook of hypnotic suggestions and metaphor (p. 523-524). New York, NY: Norton.

Wesselmann, D., \& Potter, A. E. (2009). Change in adult attachment status following treatment with EMDR: Three case studies. Journal of EMDR Practice and Research, 3(3), 178-191. http://dx.doi.org/10.1891/ 1933-3196.3.3.178

Yen, S., Shea, M. T., Battle, C. L., Johnson, D. M., Zlotnick, C., Dolan-Sewell, R., . . Mcglashan, T. H. (2002). Traumatic exposure and posttraumatic stress disorder in borderline, schizotypal, avoidant, and obsessive-compulsive personality disorders: Findings from the collaborative longitudinal personality disorders study. The Journal of Nervous and Mental Disease, 190, 510-518.

Young, J. E., Zangwill, W. M., \& Behary, W. E. (2002). Combining EMDR and schema-focused therapy: The whole may be greater than the sum of the parts. In F. Shapiro (Ed.), EMDR and the paradigm prism (p. 181-208). Washington, DC: American Psychological Association Press.

Zanarini, M. C. (2000). Childhood experiences associated with the development of borderline personality disorder. Psychiatric Clinics of North America, 23, 89-101.

Zanarini, M. C., Frankenburg, F. R., Hennen, J., Reich, D. B., \& Silk, K. R. (2004). Axis I comorbidity in patients with borderline personality disorder: 6-year follow-up 
and prediction of time to remission. The American Journal of Psychiatry, 161, 2108-2114.

Zanarini, M. C., Yong, L., Frankenburg, F. R., Hennen, J., Reich, D. B., Marino, M. F., \& Vujanovic, A. A. (2002). Severity of reported childhood sexual abuse and its relationship to severity of borderline psychopathology and psychosocial impairment among borderline inpatients. Journal of Nervous and Mental Disease, 190, 381-387.
Zimmerman, M., \& Mattia, J. I. (1999). Axis I diagnostic comorbidity and borderline personality disorder. Comprehensive Psychiatry, 40, 245-252.

Merci d'adresser toute correspondance concernant cet article à Dolores Mosquera, INTRA-TP, Instituto para el Estudio del Trauma y los Trastornos de la Personalidad, General Sanjurjo 111, $5^{\circ}$ 15006, A Coruña, Espagne. Courriel : doloresmosquera@gmail.com 